\title{
"That's in the eye of the beholder": Layers of Interpretation in Image Descriptions for Fictional Representations of People with Disabilities
}

\author{
Emory James Edwards \\ Department of Informatics, University \\ of California Irvine \\ i.edwards@uci.edu \\ Emily Blank \\ Google \\ emblank@google.com
}

\author{
Kyle Lewis Polster \\ Department of Informatics, University \\ of California Irvine \\ polsterk@uci.edu \\ Michael Gilbert \\ Google \\ mdgilbert@google.com
}

\author{
Isabel Tuason \\ Department of Informatics, University \\ of California Irvine \\ ituason@uci.edu \\ Stacy M. Branham \\ Informatics, University of California \\ Irvine \\ sbranham@uci.edu
}

\begin{abstract}
Image accessibility is an established research area in Accessible Computing and a key area of digital accessibility for blind and low vision (BLV) people worldwide. Recent work has delved deeper into the question of how image descriptions should properly reflect the complexities of marginalized identity. However, when real subjects are not available to consult on their preferred identity terminology, as is the case with fictional representations of disability, the issue arises again of how to create accurate and sensitive image descriptions. We worked with 25 participants to assess and iteratively co-design image descriptions for nine fictional representations of people with disabilities. Through nine focus groups and nineteen interviews, we discovered five key themes which we present here along with an analysis of the layers of interpretation at work in the production and consumption of image descriptions for fictional representations.
\end{abstract}

\section{CCS CONCEPTS}

- Human-centered computing $\rightarrow$ Accessibility; Empirical studies in accessibility.

\section{KEYWORDS}

Alt text, image description, representations of disability, fictional users, design system

\section{ACM Reference Format:}

Emory James Edwards, Kyle Lewis Polster, Isabel Tuason, Emily Blank, Michael Gilbert, and Stacy M. Branham. 2021. "That's in the eye of the beholder": Layers of Interpretation in Image Descriptions for Fictional Representations of People with Disabilities. In The 23rd International ACM SIGACCESS Conference on Computers and Accessibility (ASSETS '21), October 18-22, 2021, Virtual Event, USA. ACM, New York, NY, USA, 14 pages. https://doi.org/10.1145/3441852.3471222

Permission to make digital or hard copies of part or all of this work for personal or classroom use is granted without fee provided that copies are not made or distributed for profit or commercial advantage and that copies bear this notice and the full citation on the first page. Copyrights for third-party components of this work must be honored. For all other uses, contact the owner/author(s)

ASSETS '21, October 18-22, 2021, Virtual Event, USA

(C) 2021 Copyright held by the owner/author(s)

ACM ISBN 978-1-4503-8306-6/21/10.

https://doi.org/10.1145/3441852.3471222

\section{INTRODUCTION}

In 2014, the Census Bureau estimated that almost $30 \%$ of the U.S. population, or over 85 million people, had a disability [61]. Yet, people with disabilities ${ }^{1}$ are still systemically underrepresented across mainstream media. For example, a University of Southern California survey of the top 100 highest grossing movies of 2018 found that, of over 4,000 named characters or characters with speaking roles, only $1.6 \%$ were depicted with a disability [57]. Similarly, advertisements rarely include disability, and when they do, they tend to depict people with disabilities in stereotyped ways [18]. In response to rising awareness [20], representation in these and other industries is increasing, though it comes with increased responsibility to people with disabilities. This means making sure the media is itself accessible, by, for example, producing image descriptions for images of disability. It also means assuring the representations of disability themselves are not stereotypical or reductive.

The accessibility of images more generally-not just images depicting disability-has been an area of ongoing research. With increasing images and image descriptions online [27, 43], many researchers have moved to seek automated solutions (e.g., [14, 28, 65]). However, one challenge with these approaches is ensuring quality $[24,27]$. Therefore, some are working on more human-centric approaches, including the creation of guidelines based on feedback from screen reader users (e.g., [2, 49, 59]). In one such study, Bennett et al. [2] explored screen reader users' self-presentation in image descriptions and their feelings on misrepresentation of identity in human- or AI-generated image descriptions. They conclude that marginalized individuals should be the final authority on how to properly describe their identities in image descriptions. However, when an image depicts a fictional person-as is often the case with clip art, illustrations, placeholder art, or digital avatars-there is no real subject to consult, and we are left with the question: how should we describe aspects of identity such as race, gender, and disability status when the subject is fictional?

Addressing this gap, we present findings from a series of nine focus groups and nineteen interviews with people with a range of disabilities, including eleven blind or low vision (BLV) participants.

\footnotetext{
${ }^{1}$ In this paper we use a mixture of person-first and disability-first terminology in deference to the fact that language preferences are contested in different areas and subcommunities [40].
} 
Through the focus groups and interviews, we iteratively created and had participants assess image descriptions for a set of images depicting fictional disabled users. The images were commissioned from an independent artist by a large technology company, for use in their internal design system database by professional designers and developers of software systems. In this paper, we present five themes that arise from our data: disability in context, describing disability, level of detail, sites for introducing bias, and ableism in image descriptions. Drawing from our co-design and analysis, we present considerations for improved future writing of image descriptions for fictionalized representations of disability. Primarily, we identify the ways that image artists, description writers, and description readers each participate in the overall reading of the disabled representation. We examine how these layers need to be accounted for holistically, to reduce ableist bias and accessibility barriers. In light of the inability to consult with individuals depicted, as with photographic images, one of our recommendations is to engage the artist and people with lived experience of disability throughout the image and description development process.

\section{BACKGROUND AND RELATED WORK}

\subsection{Background: The Avatar Project}

This project is a collaboration between scholars at University of California, Irvine and industrial researchers and designers at Google. Prior to our collaboration, the 4th and 5th authors held a series of workshops at Google regarding how to properly represent a more diverse set of users, including users with disabilities, in various design and marketing assets. The intention of the images was to function as reusable brand-compliant components that could be used by all designers (including blind designers and developers who often lack accessible tools [60]) to represent users in company contexts. They were included in the company's design system used by designers and developers for internal documents such as mockups and personas. They would also be viewed by users in user testing environments and external advertisements. They began by using photographs of people with disabilities, who had given permission for their image to be used in such documents. However, concerns arose around branding and ethical implications of having photographs of real people used in contexts beyond their direct control. Thus, the $4^{\text {th }}$ and $5^{\text {th }}$ authors commissioned an artist to create a diverse set of 30 illustrations of fictional users.

"The Avatar Project," as the illustrations are now called, was created to help designers and engineers "think more critically about inclusivity, diversity and representation" [38]. Table 1 shows the subset of images from the Avatar Project which we assessed, alongside their image descriptions. The collection includes representations of fictionalized people of many ages, races, genders, ethnicities, professions, sexualities, disabilities, and other characteristics. There are also non-human representations, such as an illustration of a cartoon worm reading a book (Table $1 \mathrm{H})$. Six of the images included representations of visible disabilities (e.g., a skateboarder with a prosthetic arm, Table 1C) or assistive tools (e.g., a guide dog, Table 1D).

\subsection{Image Accessibility}

BLV people, especially screen reader users, require digital visual information to be rendered into text so that it can be accessed via synthesized voice or Braille display. Digital images are therefore made accessible by pairing them with textual representations. Image accessibility is an important aspect of digital accessibility broadly and is listed as the very first requirement for basic website accessibility, according to the $\mathrm{W} 3 \mathrm{C}$ [62]. There are several formats for accessible imagery [42]-including alt text, image descriptions, long descriptions, and captions. For example, a caption traditionally does not directly describe an image and is not solely or even primarily an accessibility feature. It provides supplementary information, for example artistic credit for the image [17]. An image description, unlike alt text or long description html attributes, is usually included in the body of the webpage or social media post so that screen reader users, people who use magnification, and sighted users can all make use of the image description. The content included in alt text, image descriptions, captions, and long descriptions can, but will not necessarily, overlap. In this paper we follow the convention of prior works $[2,44,59]$ by simply referring to textual image representations as "image descriptions."

Image accessibility has overall improved over the past decade, with image description prevalence on popular websites increasing from less than $40 \%$ to $72 \%$ between 2006 and 2018, respectively $[5,27]$. However, in some cases-sites like Twitter that primarily comprise user generated content-were found to have alt text on only $0.1 \%$ of images [24]. This leads to a large gap between the experiences and information available to sighted and blind consumers.

To address pervasive inaccessibility of digital imagery, the Accessible Computing field has explored several potential solutions, ranging from fully automated to highly social methods for creating and propagating image descriptions. Several approaches leverage computer vision to automatically label images [14, 28, 65]. While these deliver scalability, they cannot promise the same quality as human-generated descriptions [2, 24, 39], making automatic approaches suboptimal in some circumstances. Researchers have also explored automatic methods for finding metadata or captions provided on other instances of images and providing them to users seeking image descriptions [27]. In the realm of human-generated image descriptions, crowdsourcing [4, 10,53], friendsourcing [8, 41], and social microvolunteering [9] have all been explored as potential methods for providing image descriptions. The primary concern with human-generated methods is providing high quality information while reducing lag time between request and response. Some researchers have attempted to use multiple methods in combination to achieve high quality results [24].

To aid image description development, there are a number of guidelines detailing how to generate useful image descriptions. Some guidelines are based on feedback from BLV people and/or screen reader users [2, 39, 49, 50, 59]. There are also non-academic guidelines from a variety of sources, including museums [17], nonprofit accessibility initiatives [19], and individuals [16, 37]. Some of these guidelines deal with issues around describing a) drawings or 
Table 1: Images and Image Descriptions Assessed in Our Study

\begin{tabular}{|c|c|c|}
\hline ID & & Image Description \\
\hline A & & $\begin{array}{l}\text { A colorful 3D cartoon-like illustration of an adult with a light skin-tone and short blond hair against a red } \\
\text { background. They are smirking and wearing sunglasses, holding a white cane in one hand and a steaming coffee in } \\
\text { the other. They are wearing a sleeveless blue sports vest over a white long sleeve shirt. }\end{array}$ \\
\hline $\mathrm{B}$ & & $\begin{array}{l}\text { A colorful 3D cartoon-like illustration of an adult with dark-colored eyes and vitiligo, so that parts of their } \\
\text { medium-dark skin tone has lost pigment and is a very light brown tone, against a pale blue background. They have } \\
\text { curly hair and are wearing a green shirt and gold jewelry, looking thoughtful. }\end{array}$ \\
\hline $\mathrm{C}$ & & $\begin{array}{l}\text { A colorful 3D cartoon-like illustration of a youth with dark-colored eyes and black hair wearing a helmet and } \\
\text { holding a skateboard with a prosthetic hand and smiling against a light tan background. They have a light skin tone } \\
\text { and dark brown hair down to their shoulders. They are wearing a two-tone green shirt and have one hand tucked } \\
\text { into their red shorts. }\end{array}$ \\
\hline $\mathrm{D}$ & & $\begin{array}{l}\text { A colorful 3D cartoon-like illustration of a service dog with a green collar and silver harness, held by a pale hand. It } \\
\text { is looking sadly at an ice cream cone on the ground against a blue background. }\end{array}$ \\
\hline $\mathrm{E}$ & & $\begin{array}{l}\text { A colorful 3D cartoon-like moving } \mathrm{GIF}^{\&} \text { of an adult with long blue hair, dark-colored eyes, and light skin tone } \\
\text { against a yellow background. They are wearing a red shirt and a hearing aid. They have their fingers together and } \\
\text { hand positioned upward, resting against their chin. They motion their hand forward, smiling slightly. With this } \\
\text { motion, they are saying thank you in sign language. }\end{array}$ \\
\hline $\mathrm{F}$ & & $\begin{array}{l}\text { A colorful 3D cartoon-like illustration of an older adult with a medium skin tone, long grey hair, and dark-colored } \\
\text { eyes, wearing green glasses, a blue sari and a bindi against a yellow background. They are watering plants and } \\
\text { smiling slightly. }\end{array}$ \\
\hline G & & $\begin{array}{l}\text { A colorful 3D cartoon-like illustration of an adult with a light skin tone and a blond beard and ponytail using an } \\
\text { electric wheelchair against a pale pink background. They are wearing a green shirt and holding some groceries with } \\
\text { more hanging from their chair. They have their eyes closed and look calm and happy. }\end{array}$ \\
\hline $\mathrm{H}$ & & $\begin{array}{l}\text { A colorful 3D cartoon-like illustration of a green worm with orange glasses popping out of a hole in a red book with } \\
\text { yellow books in the background. The bookworm is reading a smaller book and smiling. }\end{array}$ \\
\hline I & & $\begin{array}{l}\text { A colorful 3D cartoon-like illustration of an interracial LGBT couple. They are each with one hand holding and } \\
\text { smiling at a baby against a pale green background. The one on the left has a medium skin tone, short dark hair, dark } \\
\text { colored eyes, and is wearing a yellow beanie. The person on the right has a light skin tone, shoulder length light } \\
\text { brown hair, dark colored eyes, and is wearing gold hoop earrings. The baby is bundled in a white blanket, resting } \\
\text { peacefully, and has a single curl of blond hair on their head. }\end{array}$ \\
\hline
\end{tabular}

\# Images created by Janet Mac and Patrick Dias [38]. \& The still version is included in this publication. The moving GIF version was the one assessed by our participants.

works of art [19] or b) photographs of people and their identities [16], but none discuss artistic depictions of disability in depth [17] or depictions of users in design contexts, both of which are relevant in our case.

In recent years, accessibility researchers have begun to investigate the complexities of writing image descriptions for BLV users in different contexts [59] and with different marginalized identities [2]. Stangl et al. [59] noted that image descriptions must respond to different contexts of use and different purposes of posting in order to accurately and sensitively describe elements of the image such as marginalized identities being depicted. Bennett et al. [2] focused on the problem of describing marginalized identities in their study of screen reader users who also had additional marginalized identities: Black, Indigenous, Person of Color, or trans or non-binary, or some combination of those labels. Along with their inquiry into participants' non-visual methods for understanding identities of people online, they found four areas where participants felt information about identity was particularly important: 1) when identity was the topic of conversation, 2) when assessing the identities being represented in media, 3) when assessing the audience or potential shared identities of people around them, and 4) when specifically seeking out the perspectives of marginalized people. Bennett et al. also discussed the limitations and potential consequences of AI-powered image descriptions, specifically the risk of misidentification, and concluded there should be more studies targeting professional content creators, rather than everyday users.

Our paper discusses similar issues and takes a similar approach to Bennett et al.'s paper, with three key distinctions, which we highlight here to frame our contributions. Firstly, our study focuses on disability identity broadly, as opposed to Bennett et al.'s indepth look at multiply marginalized screen reader users. Therefore, our recruitment did not focus solely on screen reader users, but rather included people with a variety of disabilities, both visible and invisible. Secondly, our paper looks at images created for a design context, whereas Bennett et al.'s study was primarily focused on a social media context, particularly social media where the participants were known to most of their audience. Lastly, our study assesses image descriptions of fictionalized artistic representations 
of disability, whereas Bennett et al. focused on self-presentation, particularly in photographs.

In this paper, we extend and complement prior work by exploring fictional representations of multiple disability identities in image descriptions for design contexts. Further, the guidelines generated by this study answer Bennett et al.'s call for future studies that address the problem of image descriptions in professional content creation pipelines.

\subsection{Media Representations of Disability}

Representations of disability in media is a broad topic with many facets that we can only briefly explore here. Within Media Studies and Disability Studies, there are discussions of how real people with disabilities are written about in news media and shown in advertisements $[32,52]$ and how fictional characters with disabilities are portrayed in film, television, and literature [18, 23]. New media and digital technologies are often discussed for their potential impact on and relationship with representations of disability $[25,26]$. Existing literature on representations of disability in media largely agree on two points. First, representation of disability has historically been lacking [18] and still lags behind in quantity compared to representations of minoritized genders or races [57]. Secondly, representations of disability that do exist in media can often be one-dimensional, stereotypical, patronizing, or otherwise offensive $[12,22,34]$. The ableism that shapes societal perceptions of disability and the marginalization of disabled people in the workplace and educational sphere is present also in media depictions of disability.

Written language, including image descriptions, are an important element of dismantling stereotypes. Image descriptions, specifically, have been shown to reproduce biases around identity, particularly gendered stereotypes [46, 47]. Disability advocate June Issacson Kailes wrote: "A significant element in the struggle for basic human rights is what people call themselves... Disability culture is the commonality of the experience of living with a disability, and language is one of the keys to acknowledging this culture" [35]. How disability is discussed in written media has been found to slowly adapt to changing norms of what is acceptable language [33], but how to translate visual representations of disability in media into image descriptions in a way that does not reinforce stereotypes about either disability or other marginalized identities has not been investigated yet.

The depiction of disability and other marginalized identities in design contexts is understudied. Personas, despite being a popular design and teaching tool $[29,45][25,38]$, rarely represent people with disabilities [55] or represent them in limited [21], inaccurate, or othering ways [3]. Beyond personas, there is little work on other ways users are represented in technology companies' design assets or internal materials. However, the value of increasing disability visibility in design contexts has been demonstrated in previous work on the empathetic and practical gains that can result from educating technologists about disability [51, 63].

\section{METHODS}

Our study seeks to answer the following research question: according to people with disabilities, what are important elements in defining appropriate and sensitive depictions of disability in image descriptions of fictional users? To understand this question, we conducted nine focus groups and nineteen interviews, totaling nearly 28 hours of dialogue, with 25 people who self-identified as having one or more disabilities. Our study was reviewed and approved by the Institutional Review Board (IRB) of the first author's university, and the final paper was approved through Google's internal review process. Focus groups and interviews were conducted remotely via phone, Zoom, or Skype, and participants were compensated at a rate of $\$ 20$ per hour. All sessions were video and audio recorded after obtaining written or verbal consent from participants. The first author attended all focus groups and interviews, usually with one or more additional researchers present.

\subsection{Images and Image Descriptions}

We selected a subset of nine images from the Avatar Project for assessment (Table 1). This subset included all images with representations of visible disability or assistive aids (6). We included a depiction of vitiligo (Table 1B) under the umbrella of disability representation as it is a skin condition that can impact quality of life due to stigmatization and negative attitudes [48], which is in line with the World Health Organization definition of disability [66]. We also chose to include three images without any visible disability, so we could explore the notion of invisible disability and image description. These three images depicted an older adult (Table 1F), an LGBTQ+ couple with a newborn (Table 1I), and a non-human bookworm (Table 1H). From the original two- to three-word descriptions provided by the company, and with attention to existing guidelines on image description, we generated an initial set of image descriptions that we then iteratively co-designed throughout our study. Final versions of image descriptions are presented in Table 1.

\subsection{Participants}

We recruited 25 people with disabilities (Table 2) from previous participant pools and authors' existing social networks, as well as through regional disability groups. We also used snowball sampling. We included participants based on their self-disclosure of having a disability in a pre-screening survey. We intentionally sought to recruit people who had additional marginalized racial or gender identities, to solicit thoughts regarding marginalized identities that were not disabilities.

\subsection{Focus Groups and Initial Interviews}

We conducted nine focus groups and three initial interviews using the same protocol-lasting about one hour each-to get participants' opinions and reactions to visual and written depictions of disability and other marginalized identities. We formed focus groups based on participants' availability as indicated in the initial screening survey, scheduling a focus group when at least two participants were available. The three interviews included in this section were intended to be focus groups but due to cancellations only one participant attended.

Before the start of each of these sessions, to prompt thinking about representation in images and descriptions, we asked participants to provide either a sketch or one-sentence textual description of themselves from the shoulders up. At the start of each 
Table 2: Participant IDs and Demographics

\begin{tabular}{|c|c|c|c|c|}
\hline ID & Age & Gender & Race & Disability \\
\hline $\mathrm{S} 1 \_\mathrm{P} 1(+)$ & $18-29$ & Non-binary (ve/ver/vis) & Black or African American & MIID \\
\hline $\mathrm{S} 1 \_\mathrm{P} 2$ & $18-29$ & Non-binary (they/them/theirs) & Black or African American & MIID \\
\hline $\mathrm{S} 1 \_\mathrm{P} 3(+)$ & $30-39$ & Woman & White & P, MIID, BLV ${ }^{\mathrm{i}}$ \\
\hline S1_P4(+) & $18-29$ & Man & Asian & $\mathrm{DHH}$ \\
\hline $\mathrm{S} 2 \_\mathrm{P} 1(+)$ & $18-29$ & Woman & White & $\mathrm{P}, \mathrm{MIID}^{*}$ \\
\hline $\mathrm{S} 2 \_\mathrm{P} 2(+)$ & $18-29$ & Non-binary (they/them/theirs) & Asian & MIID \\
\hline S2_P3 & $18-29$ & Non-binary (they/them/theirs) & Asian & MIID \\
\hline S3_P1 & $18-29$ & Woman & White & MIID* \\
\hline $\mathrm{S} 3 \_\mathrm{P} 2(+)$ & $30-39$ & Woman & White & $\mathrm{P}, \mathrm{MIID}$ \\
\hline $\mathrm{S} 4 \_\mathrm{P} 1(+)$ & $30-39$ & Woman & White & $\mathrm{BLV}^{\mathrm{i}}$ \\
\hline $\mathrm{S} 4 \_\mathrm{P} 2(+)$ & $18-29$ & Woman & White & MIID*, P \\
\hline S5_P1 & $40-49$ & Woman & White & $\mathrm{P}$ \\
\hline $\mathrm{S} 5 \_\mathrm{P} 2(+)$ & $30-39$ & Woman & White & $B L V \wedge$ \\
\hline S6_P1(+) & $18-29$ & Woman & White & $\mathrm{BLV} \wedge$ \\
\hline S7_P1(+) & $18-29$ & Woman & Hispanic or Latinx & $\mathrm{BLV}^{\mathrm{i}}$ \\
\hline $\mathrm{S} 8 \_\mathrm{P} 1(+)$ & $18-29$ & Woman & Black or African American & $\mathrm{P}, \mathrm{MIID}$ \\
\hline S8_P2(+) & $30-39$ & Man & White & $\mathrm{P}, \mathrm{BLV}^{\mathrm{i}}$ \\
\hline S8_P3 & $18-29$ & Woman & Asian, White & MIID \\
\hline S9_P1 & $30-39$ & Woman & Black or African American, White & DHH, BLV ${ }^{\mathrm{i}}$ \\
\hline S10_P1 & $30-39$ & Man & White & $\mathrm{BLV}^{\mathrm{i}}$ \\
\hline S10_P2 & $30-39$ & Man & White & $\mathrm{BLV}^{\mathrm{i}}$ \\
\hline $\mathrm{S} 11 \_\mathrm{P} 1(+)$ & $40-49$ & Man & Hispanic or Latinx & $\mathrm{P}$ \\
\hline $\mathrm{S} 11 \_\mathrm{P} 2(+)$ & $18-29$ & Nonbinary (they/them/theirs) & South Asian & MIID* \\
\hline $\mathrm{S} 12 \_\mathrm{P} 1(+)$ & $30-39$ & Woman & Hispanic or Latinx, White & $\mathrm{BLV}^{\mathrm{i}}$ \\
\hline $\mathrm{S} 12 \_\mathrm{P} 2(+)$ & $18-29$ & Man & White & $\mathrm{BLV}^{\mathrm{i}}$ \\
\hline
\end{tabular}

Key: DHH $=$ D/deaf or Hard of Hearing, P = Physical Disability, MIID = Mental Illness or Invisible Disability, ${ }^{*}=$ Multiple disabilities within this category, $(+)=$ Participated in a follow-up interview, ${ }^{\mathrm{i}}=$ uses screen reader some or all of the time, $\wedge=$ uses magnification

session, participants and researchers verbally shared their selfrepresentation, as researchers displayed the illustrations and/or descriptions via the teleconferencing system's screen sharing and chat features. After introductions, we presented an example interface mockup that used three avatars, to explain to participants how avatars were meant to be used by designers. Next, we displayed four to six slides, each with one avatar's illustration and its associated image description ${ }^{2}$. For the sake of time, we could only present a subset of the nine avatars in each session; our selection was such that participants would be shown avatars most closely related to their disability identity. For our participants who used screen readers, we pasted the image descriptions into the text chat as we introduced the image and gave participants time to listen to the description.

For each image description, we asked participants what they thought about the level of detail, length, accuracy, specific terms used, organization, and whether it fairly represented the identities portrayed. We also asked how they felt about the image and description being used by a potentially non-disabled technology designer. At the conclusion of the session, we asked more generally

${ }^{2}$ In session 11, no image descriptions were shown, and instead visual representation of disability was discussed. Session 11 and the follow up interviews with S11_P1 and S11_P2 are included in our analysis, as they provide general feelings about disability representation in media. about how social identifiers such as age, gender, race, and disability should be described in image descriptions; what should be considered when writing image descriptions and assessing quality; and whether invisible identities or disabilities should be included in the images or descriptions. As a form of theoretical sampling [15], we asked participants in later sessions to reflect on the participant opinions and our interpretations from earlier in the study.

\subsection{Follow-up Interviews}

We conducted follow-up interviews-lasting 30 to 60 minutes-with sixteen participants, to provide them an opportunity to elaborate on comments made in the previous session in a setting where discussing personal identities would be more comfortable. These semi-structured interviews covered what social and personal identities our participants deemed important, whether their importance changed based on certain contexts, and whether these identities informed their reaction and responses to the illustrations and image descriptions shown in the previous session. We discussed participants' experiences with representations of their disability or disability in general in text or images.

\subsection{Data Analysis}

Focus group and interview recordings were transcribed through institutionally authorized automatic transcription services and edited 
for accuracy by the researchers. The first three authors analyzed the data through line-by-line inductive open coding. After creating an initial codebook, we developed an affinity diagram using MURAL, an online collaboration tool, to form axial codes and subsequent themes. Authors met weekly during analysis to iterate on codes and themes. Though our investigation centers on fictional representations of disability in design contexts, social media was often a touchstone that participants used-implicitly or explicitly-to compare expectations of image descriptions in different contexts. We took these context switches into account during analysis.

Data analysis was informed by our collective positionality as authors. Many of us identify as having one or more marginalized identities, including invisible disabilities, but also acknowledge our privilege as designers and researchers within industry and academia. We are sensitive to not only the language used around identity, but also to the power differences when working with people with disabilities and other marginalized identities. We embrace lived experience and dialogic narrative as robust accounts for meaningmaking. Our personal identities and orientation towards justice led us to emphasize data and interpretations that did not advocate for reproducing normative assumptions about gender, race, sexuality, disability, ethnicity, or other social categories.

\section{FINDINGS}

\subsection{Disability in Context}

The context of the image and its intended purpose influenced what participants thought should be included in the image description. For disability representation specifically, disability should be foregrounded in the image description only when context and purpose demanded it.

Six participants (five BLV, 1 sighted) felt that the purpose of an image was important in deciding what to include or focus on in image descriptions. For example, S4_P2 felt that image descriptions should "essentially, prioritize the information based on... what is the photo trying to convey." Although this example does not refer to a fictional representation, we note that S4_P2 equates the purpose of the image to the image creator's intent. When discussing the decision to use gender neutral language (Table 1C), S8_P2 commented that "representing people as ambiguous is good in general, especially when it's not necessarily relevant to what you're trying to portray... The point of the image is that you're showing that amputees do things that everybody else does. . It doesn't matter what gender they are." S8_P2 felt the artist's purpose was to represent disability in a normalizing way, and thus disability should be referenced more explicitly than gender in the image description.

For eleven participants, appropriate content for image descriptions was reliant on the context-that is, the intended audience and the use cases for an image. For example, S8_P1 talked about an image description that included "curly hair" (Table 1B) and the details that are most important to her as a Black woman. She argues, "It just depends on what type of information I need to know at that moment... if it was in a hair magazine, like a Black hair magazine. . . [The reader] might want their hair like that." Similarly, S7_P1, gave social media as an example where use case may affect description content, likening the level of depth needed to "the difference between glancing at a picture and looking at one." She continued: "if you're scrolling through Facebook, you're not going to look real intensely at your friend's Facebook profile. You know what they look like." In these examples, we see how audience and use case matter. Further, the image artist's intent and the reader's familiarity with the depicted identity affect the preferred description content.

Despite mentioning that disability was personally important to their identity and how they describe themselves to others, ten participants emphasized that disability is not always contextually relevant. For example, S3_P2 described how she sometimes crops her disability out of profile images. She explained, "Sometimes I struggle. I don't typically display [my disability], but I guess If I was using an app that relates to it, then I would... I think it would be really situational." For this participant, their hesitance to include disability in some representations related directly to the platform or community she was engaging with. This insight applied to representations of both visible and invisible disability. In another example, S4_P2, who has multiple invisible disabilities, felt that when deciding how she wants to be represented, "there are nuances that are dependent on what type of site is being used. . Is it Tinder? Is this a professional site? Am I using this on my Canvas when I'm replying to my professors?" Participants generally agreed that, regardless of whether disability is visible in the image, elements of context like the audience may make it irrelevant or inappropriate to include the disability in the description.

Six participants suggested that explicitly describing disability in image descriptions could serve an educational purpose, benefitting non-disabled professionals in design contexts. S6_P1 argued that "because you're using [the avatars] for mockups, it's very important for developers to take [disability] into consideration. So, I think maybe [disability] should be front and center." S1_P1 agreed that describing disability was valuable, "to remind [designers] that these [disabled] users are going to be using [their] product.” S1_P3 believed the image descriptions would help implicitly educate designers, encouraging them to create features in their software to support image description, adding that: "companies... kind of put it on the user to [create image descriptions] ... I don't necessarily think people want to be excluding [disabled] people, you know, I just don't think they have the education around it." However, participants were careful to delineate the difference between having good representation and having good accessibility. For example, S12_P1 stated, "Good representation... [is] something more focused on taking away the stereotypes, whereas inclusive means 'let's not put a flat screen ATM machine out there." S1_P2 similarly felt that "avatars are absolutely not enough, but avatars create... awareness in society about people's disabilities." In other words, to be considered effective, good representations of disability in image descriptions had to be backed up by corporate action, in the form of accessible mainstream technologies.

\subsection{Describing Disability}

The position that disability should be foregrounded when contextually relevant but not necessarily otherwise was part of a larger theme regarding how participants talked about disability identity (their own) and how they wanted it to be talked about (in fictional representations).

Fifteen participants commented on having specific preferences for the language used to describe their own disability. For example, 
S3_P2 mentioned not feeling represented by medical language, as it did not accurately describe her experience with her disability, "I always have to look up [my condition's name], it is all medical. And then, with the condition, everyone looks completely different. There is not a 'normal' way to describe it." S4_P1 and S5_P2 mentioned feeling more connected to the "blind" community, rather than the "disability" community, more broadly. Neither very specific medical terminology nor the very broad language of "disability" felt right for these participants.

Participants' personal relationships to their disabilities often came up as factors that made them hesitant to prescribe one correct way of describing disability. All our participants disclosed that they had a disability in conversation, but in their role as image subjects, only three of the sixteen who provided image descriptions of themselves chose to mention disability in those descriptions. This may be due, in part, to the stigma associated with disability. S9_P1 said that she typically does not describe herself as blind because it has "a negative kind of connotation," and that "many people get hung up on the fact that you're blind that they don't notice anything else." Similarly, S7_P1 said: "I want to be seen, I think, as somebody, a person, before I want to be seen as a blind person." She was one of six participants in the study who explicitly stated that they did not consider or want to be portrayed as if their disability was the most important thing about them. S1_P4 mentioned a hesitation to describe his disabilities in writing, because of how it tied him to a community he didn't feel he could fully claim, "I don't feel that sense of pride over being deaf to the same degree as the other [non-mainstreamed] side of the deaf community." In contrast, S1_P3 explicitly included both her visible and invisible disabilities in her image description introduction at the beginning of the focus group. She explained in a follow-up interview that she does this with images of herself because "this was one of my ways of saying, 'okay, well these are things you might not see in the picture, but this is something I want you to know about myself in this description because it's important to my identity.", These examples show that participants have unique and complex relationships with their own disability identity, making describing disability in image descriptions a highly personal process.

Thirteen participants mentioned the importance of disability being portrayed accurately in image descriptions. S11_P1 describes how representations of disability "ha[ve] to be accurate. It is very important, because if not, you are sugarcoating it ... you don't want to sugarcoat because that is not reality." He explains how representations should not be unrealistically optimistic to the point of changing the meaning a viewer would draw from that depiction of disability. S4_P2 discussed representations of different bodies by saying, "Literally, I want to be accurate, like my body shape ... sometimes [descriptions] are like, here is someone with an hourglass figure, and that does not match how I perceive myself." Writing about disability in an idealized way, "sugarcoating it," was seen as a disservice. It functioned to make representations more palatable to non-disabled audiences, rather than accurately capturing and making visible disabled bodies.

While participants wanted accurate depictions of disabilities, this is complicated by the immense diversity, including individuals with multiple (marginalized) identities, within the disability community. For example, S11_P2 shared a desire for more, and more diverse, representations: "I think, in terms of good representation, inclusive representation doesn't exclude people.... like having as many images as possible that represent as many diverse individuals as possible." S3_P1 brought attention to the fact that the avatars with visible assistive devices in our set were all white: "It seems like it would be important to be mindful of intersections of race and ability because ... if it's always white disabled bodies next to people of color, what are the implications there? ... There's a responsibility to be careful with that." Echoing this sentiment, S1_P2 noted how creators need to be intentional when producing media, "There is a whole bunch that goes into... making art, especially trying to be representative of the whole spectrum." Accuracy, then, extends beyond the individual images to encompass the whole set; many different disabilities and distinct presentations of the same disability should be represented and shown to intersect with other forms of marginalization, such as race.

While almost all participants were comfortable discussing aspects of their own identity related to age, race, gender, and disability, they worried about or avoided definitively labeling these identities for fictional characters or images with unknown subjects. For example, S1_P3 suggested that, normally when generating image descriptions about disability, you should simply consult the subject for "permission." Similarly, S7_P1 mentioned that, in order to accurately describe her visual disability, her disability has to be "something that [the image describer and I] talked about versus making an assumption about who I am ... because everybody's eyesight is very different." S4_P2 argued that, when she reads image descriptions, she prefers that they are constructed by the subject: "It does become 'who is telling your story?'. . . because if I read it from the source of the image, I am going to feel like I have a better sense of what they want me to understand about them, [compared to] somebody who is looking at it as an observer and imposing their own biases onto the image." These examples emphasize how challenging it may be to create image descriptions for fictional avatars, where there is no access to a subject who could validate a description or give permission.

A third of participants explicitly discussed the fraught nature of labeling fictional subjects' disabilities and approved of mentioning assistive aids instead. S4_P1 felt that explicitly labeling an image subject's disability should be reserved for specific contexts such as "some kind of disability pride event... or a whole bunch of pictures of people with different disabilities." S4_P1 explained that naming assistive devices was more informative than identity labels because "it could be someone with a [service] dog or someone with a [white] cane and either way, it would be 'a blind adult."' S5_P2 and S5_P1 both agreed that describing assistive devices such as wheelchairs and white canes was preferable to using identity labels that the subject may not or cannot identify with. But even when mentioning relevant assistive devices in the image, S5_P2 clarified that one should "include the tools, but maybe not have the tools that those people use be the central focus." Even given the educational opportunity the avatars represented in the design context (Findings 4.1), participants felt that disability identity labels were so personal and complex that they hesitated to assign one to these fictional representations. Mentioning assistive devices registered to many as the correct way to represent but not overemphasize disability in image descriptions. 
These insights are not limited to disability representations; participants also expressed concern about other identities of fictional characters. For example, S2_P1 asked the interviewers if the avatars depicted real people, so there was a means to confirm their gender, to avoid misgendering them. Because the avatars refer to fictional characters, virtually all participants emphasized a need to describe visible characteristics as opposed to assuming identity labels. For example, two participants specifically used gender-neutral language such as "person" and "they" or "them" pronouns to discuss the image subjects. Instead of assuming that the people in the avatar (Table 1I) are women, S2_P3 suggested describing them as "appear[ing] to be aesthetically feminine." S3_P1 stated that describing other traits like one's clothing or accessories can allude to a person's gender as well, but also admitted this approach is based on assumptions she has about gender. Concerning race, participants largely preferred describing skin tone only, to alleviate the potential for mis-categorization. S2_P2 also believed that since race is "more of a construct," describing the subject's skin tone made the image description more "indisputably true." S10_P1, as a BLV participant, pushed back slightly on describing the skin tone. He agreed that skin tone did not always line up with race, but he felt that describing skin tone, "doesn't give me a heck of a lot of information. Whereas, at least if you say someone appears to be African American, that is much more evocative than. . . medium dark skin tone or whatever" (Table 1A). There was disagreement among participants when it came to describing identities explicitly versus avoiding assumptions by leaving wording ambiguous.

\subsection{Level of Detail}

Participants felt the right amount of detail to include in image descriptions was hard to define. There was a tension between keeping image descriptions succinct and usable, and using enough detail to convey nuance and educate readers about disability.

As might be expected, many participants mentioned that conciseness in image descriptions is important. In our study, eight participants (seven BLV, one sighted) noted that more details, especially more "irrelevant" details, can make image descriptions difficult to consume. For example, S6_P1 noted, “You don't want to have too much information in the alt text ${ }^{3}$, because then you can get overwhelmed and then it's hard to ... understand what's going on in this picture." S8_P1 also preferred clear and concise descriptions because "not everyone wants to listen or read all of that." When discussing the avatar holding a white cane and a coffee (Table 1A), S8_P2 said, "I've seen ridiculously long image descriptions ... so I'm personally perfectly fine with 'white cane'... I like economy over minute detail, unless there's some kind of reason for including minute detail.” As we will see below, the educational purpose in design contexts may constitute a reason for more detail.

Five participants mentioned how details included in image descriptions can be educational for people not familiar with that particular identity. Demonstrating the exact educational opportunity that might necessitate "minute detail," one sighted participant (S3_P1) was confused why the term "white cane" was used when

\footnotetext{
${ }^{3}$ Participants sometimes used the term "alt text" and sometimes "image description." We preserve the language they used although we solely use the term "image description" in our analysis.
}

"part of the cane is green" (Table 1A). Rather than recognizing "white cane" as the formal term of an assistive device commonly used by BLV people to navigate, she attributed the term to the color of the cane itself. The inclusion of the term "white cane" was enough to prompt questioning, but further detail in the description could have done more to educate. Similarly, when reading the description of the person with vitiligo (Table 1B), S9_P1 mentioned that she was unfamiliar with the term "vitiligo." She believed that most people would not be familiar with the term and suggested that it was more important to describe what vitiligo looks like visually rather than use the medical term. While viewing the avatar signing "Thank You" (Table 1E), a BLV participant (S10_P2) noted, "The person is clearly hard of hearing, owing to the hearing aid. You describe the motion [in the text]. So, for somebody who's reading this image, for a blind person, now they know how to say 'thank you' in sign language." We see how including more detail in image descriptions can provide a learning opportunity for readers about the disability being represented.

\subsection{Sites for Introducing Bias}

In this section, we present how participants understood image descriptions to be influenced by the author describing the imagery and the reader interpreting the description. These variables can compound on each other to create complicated differences in interpretation for the same imagery.

During the interviews, eight participants noted how image descriptions are influenced by the person who writes them and are, to a degree, subjective. S4_P2, a sighted participant, argued that image descriptions are just "another form of media... like any story, they're going to be subjective to the point of view of the person who tells it." One BLV participant (S9_P1) mentioned how "three people can look at the same picture and draw different conclusions." She went on to say that some types of subjective assumptions were especially egregious. Referencing Table 1C, she said, "don't be like, 'this picture, it is a very attractive woman.' I hate when people do that. . . be as objective as you possibly can...." Taken together, these examples suggest both an inherent subjectivity and a need to reduce bias introduced by the author's particular perspective.

Six participants (two sighted, four BLV) argued that focusing solely on visual elements could support greater objectivity. Extending the example described above, S9_P1 suggested describing the following: "What is she wearing, what is she looking at, what expression do you think is on her face? Stay away from as many adjectives as you possibly can." Similarly, S3_P1, argued that writing an image description should follow a "protocol of just writing down what you can see and not trying to infer anything more." S1_P3 said that, as image description authors, we should avoid "guessing like age or race or things like gender, [instead] keeping things just as descriptive as possible ... without saying inaccurate things." This perspective is, however, complicated by the fact that even sighted viewers may interpret the same visual facts differently. S9_P1 implied that total objectivity was likely impossible, and the goal should instead be "to be as objective as you possibly can."

Numerous examples of how the unchecked biases of image description authors can impact the BLV community surfaced in our conversations. Referring to Table 1A, S10_P2 shared, labeling someone's disability identity can be an issue, "because how would you 
know?... You're kind of insinuating that they have a 'look' about them that's blind, which a lot of visually impaired people find insulting." Including details that cannot be inferred from visuals alone, even identity labels the describer might assume are neutral, can actually reinforce biases about what blindness looks like. S9_P1 shares: "a lot of stereotypes and racism and homophobia get still passed down in the visually impaired community because you rely so... heavily on what is described to you. So, if [my father says], 'oh my god, these two women, they have this baby', you're going to have a negative connotation... because of his tone." S4_P2 distinguished between overt prejudice and more subtle bias or subjectiveness, stating, "we need to make image descriptions unbiased, in a sense that they should be free from prejudices. But I don't know that they need to be unbiased, in the sense that they're free from a sense of who the [author or subject] is or their personality" (S4_P2). Once again, there are certain types of unavoidable or benign forms of subjectivity that appear in image descriptions that can be acceptable, even while ableism, racism, homophobia, and other prejudices should not be.

One strategy participants identified to reduce bias was to seek an image description author who was familiar with the subject of the image. For example, S4_P2 explained how, "[image descriptions] speak a lot to the person who makes the image description - what they prioritize. That is why I think it's descriptions [I see on social media] done by the people who are in the pictures are oftentimes better." This implies that image descriptions produced by the subjects of images may be of better quality. But for BLV participants, they did not always have the option to independently author their own image descriptions. For example, S7_P1 relies on assistance from a sighted partner. She explains: "That [description] is basically the way that whoever you are trusting [to help create the description] sees you." In the case of fictional characters, there is no real subject of the image who can write or approve of the language used in the image description.

Beyond the biases that can appear in the writing of image descriptions, seven participants referenced the fact that reading the image description was its own type of interpretation and was influenced by their prior knowledge or experience. Upon reading an image description of an interracial couple holding a baby, S8_P2 said he assumed they were a same-sex couple even though it wasn't explicitly stated because he lives "in a certain time and place and [has] had certain experiences... but someone living. . . halfway across the world might not... it depends." S4_P2 believed that "a lot of image descriptions rely on you knowing things" and that this was intrinsic rather than something to be avoided. S4_P1 acknowledged that "how [she interpreted] the picture might not be how [she] would interpret it if [she] could see it," but she did not imply that this was a sign of a poorly written description, necessarily, just a factor in reading image descriptions in general.

Eight participants mentioned the artist or the intent behind the image as part of their final interpretation of the representation. S3_P1 referenced the "assumptions" of the image creator when discussing the imagined scenario of reverse engineering the image from a description: "if you were to use race [as opposed to skin tone] as a way to describe the images and you're using the text to create the image... that might change how in the end the image looks depending on the artist." The implication is that racial stereotypes could be reproduced in the image if an artist had stereotypical beliefs about what a certain racial identity-or presumably gender or disability identity-looks like. In addition, the author's intent was seen to also influence the final image description. One example of such thinking was provided by S8_P1: "if the artist has gone to pains to not paint the person white. . you should have some way of reflecting that in the alt text." The perspective of the artist thus cannot be excluded from those of the description authors and readers when we consider the production of inclusive descriptions of fictional avatars. These layers of interpretation-artist interpreting an identity, describer interpreting an image, reader interpreting a description-were each seen as sites where biases could be introduced into the final perceived representation.

\subsection{Ableism in Image Descriptions}

Our participants discussed the way image descriptions as a project and process are inherently oriented around a sighted worldview, and the importance of recentering people with disabilities.

Six participants described how virtual spaces are largely constructed by images, so there is a privileging of imagery over descriptive texts. In S4_P1's words, "images are definitely like a big thing that everyone uses ... [the] world that we live in is very, very vision-centric." She went on to explain, "sometimes someone might describe themselves and they might not know how they should describe themselves. They might really focus on the visual appearance of physical features ... because they have never really described themselves for someone who is blind." There is a culturally pervasive lack of skill among sighted people around describing visuals in text. Ironically, as several participants noted, sighted people are not only the predominant producers of image descriptions, but they also define what makes for 'good' image descriptions. S9_P1 explains, "because [sighted people] have the luxury of just glancing and seeing, but we don't ... [BLV people] get mired in this, 'what is important and what isn't?"' Participants argued that, while BLV readers are consumers of description, they are not given the opportunity to define what is an ideal-or even good enough-image description.

There was disagreement about how to define image descriptions as correct or complete translations of the visual image-or if visual elements even should be the gold standard for image descriptions. A total of six participants indicated that all or only visual elements should be included in image descriptions. This included three BLV participants, although all of them also voiced some support for the opposing opinion-that ambiguous or hidden aspects of the image should be articulated in descriptions-indicating that the decision is complicated. Indeed, as discussed in the section 4.4, participants suggested focusing solely on visual information and maintaining ambiguity when describing identities as a route to avoiding unnecessary or harmful biases. An example from S9_P1 brings this complexity into focus. She argued that, if race or gender of the avatars were what visually registered first for sighted people, she wanted it to be the first thing she would consume with her screen reader. "It's not important that it's a white guy or an Asian girl. But if that's what you see, then that's what you need to depict [in image descriptions]." We can see here how different readers 
advocate for different prioritization of visual cues in determining descriptions of identity.

Participants described yet another complication with limiting descriptions to what is visible in the image. Regarding disability, this would limit representation to visible disabilities and assistive technologies, leading to over-representation of visible disability and erasure of invisible disabilities. According to almost half of our participants, whether invisible disability should be mentioned in image description depended largely on the context and agency of the subject being represented. Seven of the ten participants with invisible disabilities talked about their disability in either an interview or a focus group. As discussed above, S1_P3 mentioned personally including invisible disabilities in her image descriptions even though they "are things you cannot see in a picture." She acknowledged that there is a stigma and lack of understanding associated with invisible disability and liked when it was included in descriptions because "it's hard with things like Autism to represent that in a picture, unless you put like an infinity symbol in it." In contrast, S6_P1 shared that she avoids displaying her disability in images because, "I think it is good to present more normal ... because when you see a picture [of visible disability], that would stand out." The overemphasis on visual elements as the defining factor for what is important to include in textual descriptions means that visible disabilities are seen as very distinguishing, while invisible disabilities are not represented at all.

\section{DISCUSSION}

The Accessible Computing community has been developing methods and guidelines for increased image accessibility for well over a decade [5, 49]. In recent years, studies have focused on the complicated question of how to properly depict social identities in image descriptions [2,59]. Researchers have persuasively argued for the value of sensitively describing identity in both human-centric image accessibility processes and computer-vision based identity labelling $[2,54]$. We build directly on Bennett et al.'s foundational study of self-presentation in descriptions to investigate descriptions of disability in artistic media. We provide the first study of fictional representations of disability in image descriptions. Below, we summarize considerations resulting from our findings (Table 3) and articulate three takeaways from our work: the effects of layered interpretation on image description reception, the need to displace ocularcentrism with holistic representations of identity, and the competing needs at play when depicting disability in descriptions.

\subsection{Effects of Layered Interpretation on Image Description Reception}

Bennett et al.s study looked at the challenges and opportunities of representing marginalized identity in image descriptions [2]. They primarily focused on self-presentation in photography and argued that the subject of a photograph should be the final authority on how to describe their identities. Our findings complicate this guidance. Our participants agreed that, when possible, the subject of an image should be consulted about how to properly label their identities. However, in cases such as ours where subjects are fictional and cannot be consulted, participants instead wanted to keep assumptions about identities to a minimum. As seen in section
4.2, they supported using gender-neutral language, describing skin tone instead of assuming race, and including assistive devices rather than labeling disabilities. Many of our participants, both blind and sighted, did not feel comfortable projecting-or suggesting image describers should project-identities onto fictional characters that they did not believe were visually verifiable. The interpretations of a sighted image description author were not considered to be an adequate authority on what identities were displayed or the construction of the description, even in the case of a fictional character. Media studies has engaged deeply with the concept of reader response theory-the idea that "meaning does not reside in the text, but in the mind of the reader" [67]. However, for our participants, in the situation where description authors sit between the media and the reader, in contexts as potentially fraught as disability identity representation in design, assuming and labeling even fictional representations should not be left to sighted description authors. This suggests to us an alternative source of authority as regards the identity depicted in the fictional representation: the image author.

Our findings thus reveal the layers of interpretation at work in the final reception of an image description. There are at least three layers at work in our specific case of the avatar project. First, an artist renders a fictional interpretation of disability or any other social identity. Then, the image describer interprets the fictional subject into text. Finally, the reader of the description interprets the text. In our data, we saw that the identities of the "interpreters" at each stage of the translation layered on their own biases, assumptions, or perceived meanings. This framing is an intentional echo of foundational media studies concepts about the process by which media consumers "decode" the messages that are "encoded" by media producers [31]. However, applying these media studies concepts to image accessibility reveals something new: there can be no last-minute software patch or crowdsourced intermediary that can make a fictional representation or even its image description be received as inclusive by readers. Instead, if we want image descriptions to be received, ultimately, as inclusive, there must supports throughout the interpretation pipeline. From image author, to describer, to reader, the interpretations must line up to create a final, inclusive representation of disability in the mind of the reader.

For Accessible Computing, we see the pragmatic implication to be that post-hoc creation of image descriptions cannot generate inclusive experiences for users without having accessibility built into the content creation pipeline from the beginning. While many image accessibility solutions take the form of plug-ins, extensions, or other client-side attempts to provide information, we would suggest that more inclusive experiences could be achieved by having people with disabilities, as well as other marginalized identities, involved at all stages of media creation. If artists, designers, corporations, and casual image posters can be prompted to think more deeply about disability from the beginning then image descriptions will be just one piece of considering accessibility and inclusivity. And if, as Bennett et al. suggest, marginalized identities should be described by those with the authority to do so, artists should ideally be writing their own descriptions, or they should be consulted when creating descriptions of their fictional representations. 
Table 3: Summarized Considerations for Future Image Description Creation

\begin{tabular}{|c|c|}
\hline $\begin{array}{l}\text { Who } \\
\text { imag }\end{array}$ & $\begin{array}{l}\text { If possible, construct image descriptions with the help of actors who are familiar with the subject matter in } \\
\text { review (4.4) } \\
\text { If the image describer is not the image artist, work in partnership with the artist to understand an image's } \\
\text { motivation (4.4) }\end{array}$ \\
\hline $\begin{array}{l}\text { Where is the image } \\
\text { situated? }\end{array}$ & $\begin{array}{l}\text { Consider the setting the image will exist in (ex. Digital magazine) and if a description of disability will be } \\
\text { relevant (4.1) } \\
\text { Consider the purpose of the image when constructing image descriptions (4.1) } \\
\text { Provide extra detail when images serve an educational purpose, but avoid irrelevant details (4.5) }\end{array}$ \\
\hline $\begin{array}{l}\text { How do you describe } \\
\text { visual elements? }\end{array}$ & $\begin{array}{l}\text { If disability is relevant and important to the image, describe specific attributes or communities, not 'disability' } \\
\text { generally ( } 4.2 \text { ) } \\
\text { Consider describing assistive devices instead of a subject's disability directly, to avoid assuming identities (4.2) }\end{array}$ \\
\hline $\begin{array}{l}\text { How do you avoid } \\
\text { misrepresentations? }\end{array}$ & $\begin{array}{l}\text { Be aware of existing stereotypes and consequences of misrepresenting disability in various contexts (4.1) } \\
\text { Consider the greater narrative of the individual being represented, and what describing their gender, race, or } \\
\text { other identities contributes to the image's description (4.2) } \\
\text { Avoid describing irrelevant visual details as to avoid ocularcentrism (4.5) } \\
\text { Use careful language to avoid over-emphasizing disability and to avoid altering the intended meaning of an } \\
\text { image (4.2) }\end{array}$ \\
\hline
\end{tabular}

\subsection{Ocularcentrism and Non-Visual Representations of Disability in Descriptions}

Our findings also form a second bridge between Accessible Computing and studies of disability in media. Disability Studies scholar David Bolt has found that even advertisements that depict blindness-presumably intended for a blind audience-portray disability in distorted, othering, reductive, and inaccessible ways [7]. He has discussed this as a form of "ocularcentrism" or "sighted supremacy" that demonstrates the consistent prioritization of sighted consumers' ableist worldview over the lived experience of blind people [6]. Ocularcentrism has been found to contribute to societal, personal, and internalized stigmatization among blind people [13]. What our findings (section 4.5) demonstrate is that even when people with disabilities are discussing something as inherently inclusive as image accessibility, there are elements of ocularcentrism at play. Participants were aware that image descriptions were part of a fundamentally image-centric digital environment. BLV people are the primary audience for image descriptions and several participants agreed that non-visual information also had a place in image descriptions (section 4.1). And yet it was an oftenunquestioned assumption that image descriptions should either function as very crude translations of the basic idea of the image, or that as exact replicas of even the most minute visual details. In either case, underlying these ideas is the ableist and ocularcentrist notion that image descriptions cannot be an artform in and of themselves. Image description can be art, poetry in and of itself [68], not simply seen as an imitation of the "true" (i.e., sighted) experience.

Accessible Computing is just beginning to grapple with ocularcentrism in its study of image accessibility. Bennett et al.'s work makes the point that screen reader users have many non-visual methods to gather information and make judgements about appearance [2]. Similarly, there is a growing body of work within Accessible Computing focused on novel or multisensory methods for making images accessible [36, 42, 58, 64]. Our findings suggest that another avenue for study may be the intentional representation of non-visual qualities or identities such as invisible disabilities. Consulting with disabled and otherwise marginalized image authors and media creators may allow us to create fundamentally accessible and inclusive experiences [1] that do not center a sighted or ableist perspective.

\subsection{Competing Needs When Depicting Disability in Descriptions}

One of the difficulties apparent in our data are the competing access needs $[11,30]$ at play in describing disability. Because our participants identified as having a range of disability, racial, and gender identities, we found that there was no one image description for any given avatar to best support them all. Moreover, sometimes the needs of people with one identity conflicted with those of another. For example, referring to Table 1A, S8_P1 noted that, as a BLV participant, he preferred the "economy" of a description simply using the term "white cane." However, S3_P1, a sighted participant, was unfamiliar with the term "white cane" and the lack of a detailed definition of the assistive device meant she could not properly interpret the image. We might interpret this as a missed opportunity to educate someone with a different disability identity. So, when we think back to the intent of the images in the Avatar Project, there is a tension between creating detailed image descriptions that educate primarily non-disabled designers and more concise image descriptions that serve the representational access needs of people with disabilities.

In light of these findings, Accessible Computing scholars should consider the ways that all image descriptions ultimately must balance the informational and representational of readers with different identities. For example, as crowdsourced methods for image accessibility continue to flourish $[53,56]$, attention should be paid to the often-overlooked decisions that get made by describers about identity terminology and labeling. Looking back at-in determining how to put disability in context, describe disability, select level of 
detail, and introduce degrees of bias-we see how image descriptions may be shaped by competing access. Future guidelines for image describers may benefit from explicitly discussing these competing access needs and how to navigate them in different contexts, including design.

\section{LIMITATIONS AND FUTURE WORK}

This work is one piece of an ongoing project investigating images and accessibility in design contexts. This includes interviewing artists on image construction, designers on image uses and processes of accessibility, and ongoing studies on ways of creating accessible imagery depicting disability. In addition to the limited nature of this paper as part of a larger investigation, there are several limitations arising from the specific context and design of this study. The impressions participants formed of the images and descriptions were informed by our explanation of the design context. Our findings support the idea that future studies are needed to assess media representations of disability created for other purposes or encountered in other contexts. We also recommend further studies explore in depth the requirements for depicting specific disabilities in image descriptions for fictional representations of disability.

Another limitation of the study is the limited pool of identities represented by our participants. While we had some diversity in terms of gender and race, our sample included more white people than is an accurate proportion of the general population. We also did not have any older adults who participated in the study, which meant we had a dearth of data related to age and its intersection with disability, even though older adults form a large percentage of the disability population at large [61]. We engaged with participants who were fluent in English and were based in or had emigrated from the contiguous United States. Results are thus limited to a Western, Anglophone context. Further research on image description practices in other languages and cultural contexts is needed.

Lastly, much of our stated goal for this project is based on the underlying assumption that non-disabled designers can and should be encouraged to consider and empathize with disabled users. While the authors of this piece still believe in the core promise of empathy, we respect the problems with personas and other empathy-based tools that other accessibility researchers have highlighted [3]. We hold in tension the desire for designers to put in the time and effort to make deep connections with disabled people [3] and the pragmatic belief that educating non-disabled designers in small ways can affect positive change. We encourage further work into representations of disabled users in design contexts, as well as more active models to educate corporate technologists about disabilities.

\section{CONCLUSION}

We have presented our study of the creation and assessment of image descriptions for fictional representations of disability. We situate our work in terms of Accessible Computing's study of image accessibility and literature on media representations of disability. We were especially motivated and are in debt to Bennett et al.'s paper on self-presentation of marginalized identity in image descriptions [2]. Our own study consisted of nine focus groups and nineteen follow-up interviews with 25 people with disabilities and an iterative process of image description co-design. Arising from these data, we discussed five key themes: disability in context, describing disability, level of detail, sites for introducing bias, and ableism in image descriptions. We contribute an analysis of the layers of interpretation involved in the creation and consumption of image descriptions for fictional representations of disability. We also discuss ocularcentrism and competing needs to consider in future studies of image accessibility.

\section{ACKNOWLEDGMENTS}

Thank you so much to Cella Monet Sum, without whom this project would not have made it this far. Thank you also to our participants and the ASSETS reviewers for helping us make this paper what it is today.

\section{REFERENCES}

[1] Meryl Alper. 2021. Critical Media Access Studies: Deconstructing Power, Visibility, and Marginality in Mediated Space. International Journal of Communication 15, (2021), 840-861.

[2] Cynthia L. Bennett, Cole Gleason, Morgan Klaus Scheuerman, Jeffrey P. Bigham, Anhong Guo, and Alexandra To. 2021. "It's Complicated": Negotiating Accessibility and (Mis)Representation in Image Descriptions of Race, Gender, and Disability. In Proceedings of the 2021 CHI Conference on Human Factors in Computing Systems, ACM, Yokohama Japan, 1-19. DOI:https://doi.org/10.1145/3411764.3445498

[3] Cynthia L. Bennett and Daniela K. Rosner. 2019. The Promise of Empathy: Design, Disability, and Knowing the "Other." In Proceedings of the 2019 CHI Conference on Human Factors in Computing Systems (CHI '19), ACM, New York, NY, USA, 298:1-298:13. DOI:https://doi.org/10.1145/3290605.3300528

[4] Jeffrey P. Bigham, Chandrika Jayant, Hanjie Ji, Greg Little, Andrew Miller, Robert C. Miller, Robin Miller, Aubrey Tatarowicz, Brandyn White, and Samual White. 2010. VizWiz: nearly real-time answers to visual questions. In Proceedings of the 23nd annual ACM symposium on User interface software and technology, 333-342.

[5] Jeffrey P. Bigham, Ryan S. Kaminsky, Richard E. Ladner, Oscar M. Danielsson, and Gordon L. Hempton. 2006. WebInSight:: making web images accessible. In Proceedings of the 8th international ACM SIGACCESS conference on Computers and accessibility - Assets '06, ACM Press, Portland, Oregon, USA, 181. DOI:https: //doi.org/10.1145/1168987.1169018

[6] David Bolt. 2014. The supremacy of sight: Aesthetics, representations, and attitudes. In Changing social attitudes toward disability: perspectives from historical, cultural, and educational studies. New York: Routledge, Taylor \& Francis Group, London.

[7] David Bolt. 2014. An advertising aesthetic: Real beauty and visual impairment. British Journal of Visual Impairment 32, 1 (January 2014), 25-32. DOI:https: //doi.org/10.1177/0264619613512619

[8] Erin L. Brady, Yu Zhong, Meredith Ringel Morris, and Jeffrey P. Bigham. 2013. Investigating the appropriateness of social network question asking as a resource for blind users. In Proceedings of the 2013 conference on Computer supported cooperative work - CSCW'13, ACM Press, San Antonio, Texas, USA, 1225. DOI:https://doi.org/10.1145/2441776.2441915

[9] Erin Brady, Meredith Ringel Morris, and Jeffrey P. Bigham. 2015. Gauging receptiveness to social microvolunteering. In Proceedings of the 33rd Annual ACM Conference on Human Factors in Computing Systems, 1055-1064.

[10] Erin Brady, Meredith Ringel Morris, Yu Zhong, Samuel White, and Jeffrey P. Bigham. 2013. Visual challenges in the everyday lives of blind people. In Proceedings of the SIGCHI Conference on Human Factors in Computing Systems (CHI '13), Association for Computing Machinery, New York, NY, USA, 2117-2126. DOI:https://doi.org/10.1145/2470654.2481291

[11] Stacy M. Branham and Shaun K. Kane. 2015. Collaborative Accessibility: How Blind and Sighted Companions Co-Create Accessible Home Spaces. In Proceedings of the 33rd Annual ACM Conference on Human Factors in Computing Systems (CHI '15), ACM, New York, NY, USA, 2373-2382. DOI:https://doi.org/10.1145/2702123. 2702511

[12] Emma Briant, Nick Watson, and Gregory Philo. 2013. Reporting disability in the age of austerity: the changing face of media representation of disability and disabled people in the United Kingdom and the creation of new 'folk devils.' Disability \& Society 28, 6 (September 2013), 874-889. DOI:https://doi.org/10.1080/ 09687599.2013.813837

[13] Laura Yvonne Bulk, Andrea Smith, Laura Nimmon, and Tal Jarus. 2020. A closer look at opportunities for blind adults: Impacts of stigmatization and ocularcentrism. British fournal of Visual Impairment 38, 3 (September 2020), 270-283. DOI:https://doi.org/10.1177/0264619620911424

[14] Vítor Carvalho and Diamantino Freitas. 2015. Automatic Description of SVG Images for the Visually Impaired: A Gestaltic Approach. Procedia Computer 
Science 67, (2015), 2-11. DOI:https://doi.org/10.1016/j.procs.2015.09.243

[15] Kathy Charmaz. 2014. Constructing grounded theory. sage.

[16] Alex Chen. 2020. How to write an image description. Medium. Retrieved March 14 2021 from https://uxdesign.cc/how-to-write-an-image-description-2f30d3bf5546

[17] Cooper Hewitt Smithsonian Design Museum. 2019. Cooper Hewitt Guidelines for Image Description. Cooper Hewitt Smithsonian Design Museum. Retrieved August 8, 2020 from https://www.cooperhewitt.org/cooper-hewitt-guidelinesfor-image-description/

[18] Lennard J. Davis. 2013. The disability studies reader (4th ed ed.). Routledge, New York, NY.

[19] DIAGRAM Center. 2019. Image Description Guidelines. Retrieved March 14, 2021 from http://diagramcenter.org/table-of-contents-2.html/

[20] Tom DiChristopher. 2015. Fans to Netflix: Make Daredevil accessible to the blind. CNBC. Retrieved March 12, 2021 from https://www.cnbc.com/2015/04/10/fansto-netflix-make-daredevil-accessible-to-the-blind.html

[21] Emory James Edwards, Cella Monet Sum, and Stacy M. Branham. 2020. Three Tensions Between Personas and Complex Disability Identities. In Extended $A b-$ stracts of the $2020 \mathrm{CHI}$ Conference on Human Factors in Computing Systems (CHI EA '20), Association for Computing Machinery, New York, NY, USA, 1-9. DOI:https://doi.org/10.1145/3334480.3382931

[22] Katie Ellis, Debbie Rodan, and Pia Lebeck. Disability, Obesity and Ageing. 177.

[23] Rosemarie Garland-Thomson. 2017. Extraordinary bodies: figuring physical disability in American culture and literature (Twentieth anniversary edition ed.). Columbia University Press, New York.

[24] Cole Gleason, Amy Pavel, Emma McCamey, Christina Low, Patrick Carrington, Kris M. Kitani, and Jeffrey P. Bigham. 2020. Twitter A11y: A Browser Extension to Make Twitter Images Accessible. In Proceedings of the 2020 CHI Conference on Human Factors in Computing Systems, ACM, Honolulu HI USA, 1-12. DOI:https //doi.org/10.1145/3313831.3376728

[25] Gerard Goggin, Katie Ellis, and Wayne Hawkins. 2019. Disability at the centre of digital inclusion: assessing a new moment in technology and rights Communication Research and Practice 5, 3 (July 2019), 290-303. DOI:https: //doi.org/10.1080/22041451.2019.1641061

[26] Gerard Goggin and Christopher Newell. 2007. The Business of Digital Disability. The Information Society 23, 3 (April 2007), 159-168. DOI:https://doi.org/10.1080/ 01972240701323572

[27] Darren Guinness, Edward Cutrell, and Meredith Ringel Morris. 2018. Caption Crawler: Enabling Reusable Alternative Text Descriptions using Reverse Image Search. In Proceedings of the 2018 CHI Conference on Human Factors in Computing Systems - CHI '18, ACM Press, Montreal QC, Canada, 1-11. DOI:https://doi.org/ $10.1145 / 3173574.3174092$

[28] Ankush Gupta and Prashanth Mannem. 2012. From Image Annotation to Image Description. In Neural Information Processing, Tingwen Huang, Zhigang Zeng, Chuandong Li and Chi Sing Leung (eds.). Springer Berlin Heidelberg, Berlin, Heidelberg, 196-204. DOI:https://doi.org/10.1007/978-3-642-34500-5_24

[29] Maren Haag and Nicola Marsden. 2019. Exploring personas as a method to foster empathy in student IT design teams. Int 7 Technol Des Educ 29, 3 (May 2019), 565-582. DOI:https://doi.org/10.1007/s10798-018-9452-5

[30] Oliver L. Haimson and Anna Lauren Hoffmann. 2016. Constructing and enforcing "authentic" identity online: Facebook, real names, and non-normative identities First Monday 21, 6 (June 2016). DOI:https://doi.org/10.5210/fm.v21i6.6791

[31] Stuart Hall. 2003. Encoding/decoding. Television: critical concepts in media and cultural studies 4, (2003), 43-53.

[32] Beth A. Haller. Representing Disability in an Ableist World.

[33] Beth Haller, Bruce Dorries, and Jessica Rahn. 2006. Media labeling versus the US disability community identity: a study of shifting cultural language. Disability \& Society 21, 1 (January 2006), 61-75. DOI:https://doi.org/10.1080/ 09687590500375416

[34] Ella Houston. 2019. 'Risky' representation: the portrayal of women with mobility impairment in twenty-first-century advertising. Disability \& Society 34, 5 (May 2019), 704-725. DOI:https://doi.org/10.1080/09687599.2019.1576505

[35] June Isaacson Kailes. 1985. Watch your language, please! fournal of Rehabilitation 51, 1 (1985), 68.

[36] Nahyun Kwon, Youngji Koh, and Uran Oh. 2019. Supporting Object-level Exploration of Artworks by Touch for People with Visual Impairments. In The 21st International ACM SIGACCESS Conference on Computers and Accessibility (ASSETS '19), Association for Computing Machinery, New York, NY, USA, 600-602. DOI:https://doi.org/10.1145/3308561.3354620

[37] Veronica Lewis. 2018. How To Write Alt Text And Image Descriptions For The Visually Impaired. Veroniiiica. Retrieved August 8, 2020 from https://veroniiiica. com/2018/01/31/how-to-write-alt-text-image-descriptions-visually-impaired/

[38] Janet Mac. 3D Illustration \& Animation - Google Project. Retrieved April 13, 2021 from https://janet-mac.com/google-avatar-project

[39] Haley MacLeod, Cynthia L. Bennett, Meredith Ringel Morris, and Edward Cutrell. 2017. Understanding Blind People's Experiences with Computer-Generated Captions of Social Media Images. In Proceedings of the 2017 CHI Conference on Human Factors in Computing Systems, ACM, Denver Colorado USA, 5988-5999. DOI:https://doi.org/10.1145/3025453.3025814
[40] Jennifer Mankoff, Gillian R. Hayes, and Devva Kasnitz. 2010. Disability studies as a source of critical inquiry for the field of assistive technology. In Proceedings of the 12th international ACM SIGACCESS conference on Computers and accessibility, ACM, 3-10.

[41] Reeti Mathur and Erin Brady. 2018. Mixed-Ability Collaboration for Accessible Photo Sharing. In Proceedings of the 20th International ACM SIGACCESS Conference on Computers and Accessibility (ASSETS '18), Association for Computing Machinery, Galway, Ireland, 370-372. DOI:https://doi.org/10.1145/3234695.3240994

[42] Meredith Ringel Morris, Jazette Johnson, Cynthia L. Bennett, and Edward Cutrell. 2018. Rich Representations of Visual Content for Screen Reader Users. In Proceedings of the 2018 CHI Conference on Human Factors in Computing Systems CHI '18, ACM Press, Montreal QC, Canada, 1-11. DOI:https://doi.org/10.1145/ 3173574.3173633

[43] Meredith Ringel Morris, Annuska Zolyomi, Catherine Yao, Sina Bahram, Jeffrey P. Bigham, and Shaun K. Kane. 2016. "With most of it being pictures now, I rarely use it”: Understanding Twitter's Evolving Accessibility to Blind Users. In Proceedings of the 2016 CHI Conference on Human Factors in Computing Systems, ACM, San Jose California USA, 5506-5516. DOI:https://doi.org/10.1145/2858036.2858116

[44] Julius T. Nganji, Mike Brayshaw, and Brian Tompsett. 2013. Describing and Assessing Image Descriptions for Visually Impaired Web Users with IDAT. In Proceedings of the Third International Conference on Intelligent Human Computer Interaction (IHCI 2011), Prague, Czech Republic, August, 2011, Miloš Kudělka, Jaroslav Pokorný, Václav Snášel and Ajith Abraham (eds.). Springer Berlin Heidelberg, Berlin, Heidelberg, 27-37. DOI:https://doi.org/10.1007/978-3-642-31603-6_3

[45] Lene Nielsen. 2013. Personas-user focused design. Springer.

[46] Jahna Otterbacher. 2015. Crowdsourcing Stereotypes: Linguistic Bias in Metadata Generated via GWAP. In Proceedings of the 33rd Annual ACM Conference on Human Factors in Computing Systems, ACM, Seoul Republic of Korea, 1955-1964. DOI:https://doi.org/10.1145/2702123.2702151

[47] Jahna Otterbacher, Pınar Barlas, Styliani Kleanthous, and Kyriakos Kyriakou. 2019. How Do We Talk about Other People? Group (Un)Fairness in Natural Language Image Descriptions. HCOMP 7, 1 (October 2019), 106-114.

[48] Davinder Parsad, Sunil Dogra, and Amrinder Jit Kanwar. 2003. Quality of life in patients with vitiligo. Health and Quality of Life Outcomes (2003), 3.

[49] Helen Petrie, Chandra Harrison, and Sundeep Dev. 2005. Describing images on the web: a survey of current practice and prospects for the future. Proceedings of Human Computer Interaction International (HCII) 71, (2005), 2.

[50] Christopher Power. 2012. Guidelines are only half of the story: accessibility problems encountered by blind users on the web. (2012), 10.

[51] Cynthia Putnam, Maria Dahman, Emma Rose, Jinghui Cheng, and Glenn Bradford. 2015. Teaching Accessibility, Learning Empathy. In Proceedings of the 17th International ACM SIGACCESS Conference on Computers \& Accessibility (ASSETS '15), ACM, New York, NY, USA, 333-334. DOI:https://doi.org/10.1145/2700648.2811365

[52] Charles A. Riley. 2005. Disability and the media: prescriptions for change. University Press of New England, Hanover, $\mathrm{NH}$.

[53] Elliot Salisbury, Ece Kamar, and Meredith Ringel Morris. 2017. Toward Scalable Social Alt Text: Conversational Crowdsourcing as a Tool for Refining Vision-toLanguage Technology for the Blind. Proceedings of the Fifth AAAI Conference on Human Computation and Crowdsourcing (2017), 10.

[54] Morgan Klaus Scheuerman, Kandrea Wade, Caitlin Lustig, and Jed R. Brubaker. 2020. How We've Taught Algorithms to See Identity: Constructing Race and Gender in Image Databases for Facial Analysis. Proc. ACM Hum.-Comput. Interact. 4, CSCW1 (May 2020), 1-35. DOI:https://doi.org/10.1145/3392866

[55] Trenton Schulz and Kristin Skeide Fuglerud. 2012. Creating Personas with Disabilities. In Computers helping people with special needs: 13th International Conference, ICCHP 2012, Linz, Austria, fuly 11-13, 2012: proceedings, Klaus Miesenberger, Arthur Karshmer, Petr Penaz and Wolfgang Zagler (eds.). Springer, Berlin; New York.

[56] Rachel N. Simons, Danna Gurari, and Kenneth R. Fleischmann. 2020. "I Hope This Is Helpful”: Understanding Crowdworkers' Challenges and Motivations for an Image Description Task. Proc. ACM Hum.-Comput. Interact. 4, CSCW2 (October 2020), 105:1-105:26. DOI:https://doi.org/10.1145/3415176

[57] Dr Stacy L Smith, Marc Choueiti, and Dr Katherine Pieper. 2020. Inequality in 1,300 Popular Films: Examining Portrayals of Gender, Race/Ethnicity, LGBTQ \& Disability from 2007 to 2019. Annenburg Inclusion Initiative, USC.

[58] Leandro Soares Guedes. 2021. Designing multisensory experiences for users with different reading abilities visiting a museum. SIGACCESS Access. Comput. 129 (March 2021), 3:1-3:6. DOI:https://doi.org/10.1145/3458055.3458058

[59] Abigale Stangl, Meredith Ringel Morris, and Danna Gurari. 2020. "Person, Shoes, Tree. Is the Person Naked?" What People with Vision Impairments Want in Image Descriptions. In Proceedings of the 2020 CHI Conference on Human Factors in Computing Systems, ACM, Honolulu HI USA, 1-13. DOI:https://doi.org/10. 1145/3313831.3376404

[60] Kevin M Storer, Harini Sampath, and M. Alice Alice Merrick. 2021. "It's Just Everything Outside of the IDE that's the Problem":: Information Seeking by Software Developers with Visual Impairments. In Proceedings of the $2021 \mathrm{CHI}$ Conference on Human Factors in Computing Systems, ACM, Yokohama Japan, 1-12. DOI:https://doi.org/10.1145/3411764.3445090 
[61] Danielle M. Taylor. 2018. Americans With Disabilities: 2014. U.S. Census Bureau, United States. Retrieved from https://www.census.gov/library/publications/2018/ demo/p70-152.html

[62] W3C Web Accessibility Initiative. 2019. How to Meet WCAG (Quickref Reference) Retrieved April 9, 2021 from https://www.w3.org/WAI/WCAG21/quickref/\#top

[63] Annalu Waller, Vicki L. Hanson, and David Sloan. 2009. Including accessibility within and beyond undergraduate computing courses. In Proceeding of the eleventh international ACM SIGACCESS conference on Computers and accessibility - ASSETS '09, ACM Press, Pittsburgh, Pennsylvania, USA, 155. DOI:https://doi.org/10.1145/ 1639642.1639670

[64] R. Michael Winters, Neel Joshi, Edward Cutrell, and Meredith Ringel Morris. 2019. Strategies for Auditory Display of Social Media. Ergonomics in Design 27, 1
(January 2019), 11-15. DOI:https://doi.org/10.1177/1064804618788098

[65] Shaomei Wu, Jeffrey Wieland, Omid Farivar, and Julie Schiller. 2017. Automatic Alt-text: Computer-generated Image Descriptions for Blind Users on a Social Network Service. In Proceedings of the 2017 ACM Conference on Computer Supported Cooperative Work and Social Computing, ACM, Portland Oregon USA, 1180-1192. DOI:https://doi.org/10.1145/2998181.2998364

[66] Disability and health. Retrieved April 8, 2021 from https://www.who.int/newsroom/fact-sheets/detail/disability-and-health

[67] reader-response theory. Oxford Reference. DOI:https://doi.org/10.1093/oi/ authority.20110803100406762

[68] Alt-Text as Poetry. Retrieved April 13, 2021 from https://alt-text-as-poetry.net 